\title{
ANALYZING THE DISTRIBUTION OF DECAY CONSTANTS IN PULSE-FLUORIMETRY USING THE MAXIMUM ENTROPY METHOD
}

\author{
A. K. Livesey AND J. C. Brochon \\ Laboratoire pour l'Utilisation du Rayonnement Electromagnétique, Centre National de la Recherche \\ Scientifique/Commissariat à l'Energie Atomique, Ministère de l'Education Nationale, Université de \\ Paris-Sud, F 91405 Orsay Cedex, France
}

\begin{abstract}
The maximum entropy method (MEM) is used to analyze time-resolved pulse-fluorescence spectrometry. The central problem in such analyses is the recovery of the distribution of exponentials describing the decay of the fluorescence (i.e., inverting the Laplace transform) which is, in turn, convolved by the shape of the excitation flash. MEM is shown to give high quality results from both computer-generated "noisy" data and experimental data from chemical and biological molecules.

The use of the Shannon-Jaynes entropy function is justified and both the theoretical and practical advantages of MEM are presented. The MEM results are easy to interpret and can help to overcome some experimental limitations. In particular MEM could be a powerful tool to analyze the heterogeneity of fluorescent emission of biological macromolecules which can be correlated with their conformational dynamics in solution.
\end{abstract}

\section{INTRODUCTION}

Fluorescence spectroscopy has now become a classical method for studying biological molecules in solution. However, steady-state measurements, even though they are very sensitive, cannot probe the molecular origins of the observed effect. The excited states of the fluorophores have a half-life in the range of a few picoseconds to some tens of nanoseconds. Since this corresponds to the timescale of many important biological processes (diffusion of small molecules over a few angstroms, rotational and internal motions, proton transfer reactions, etc., time-resolved fluorometry has become an important and popular tool. Various pulse fluorometric techniques can measure such short decay times with good precision and of these the singlephotoelectron time-correlated method is now the most sensitive (Ware, 1971; Yguerabide, 1972; Wahl, 1975).

Despite the great advances in measurement techniques over recent years, the analysis of the data is fraught with difficulties. The fluorescent kinetics are usually very complicated arising from the heterogeneity of the types of fluorophores and from their varied physical or chemical environments. Furthermore, the core of the problem is a

Address correspondence to J. C. Brochon.

Dr. Livesey's current address is Department of Applied Mathematics and Theoretical Physics, University of Cambridge, Silver St., MRC, Addenbrookes Site, Hills Road, Cambridge, UK.
Laplace transform, which is notorious for the ill-conditioning it introduces into problems.

The maximum entropy method (MEM) is a powerful data analysis technique, which has been justifiably used in applications as diverse as image analysis, radio astronomy, forensic science, crystallography, medical imaging, etc. (for a review see Gull and Skilling, 1984a, b). In early work on applying MEM to the Laplace transform, Sibisi (1983a) was, however, pessimistic about its ability to handle such data, primarily because he considered a mathematical example with a rather unrealistically small set of data points. Nevertheless, by including the Laplace transform as a second dimension in the analysis of nuclear magnetic resonance (NMR) data, he was able to improve radically the resolution along the frequency axis (Sibisi, $1983 b$ ).

We demonstrate here that MEM can successfully handle Laplace transforms such as those found in pulsefluorometry, without restricting the validity of the solution or suffering from any instabilities.

For clarity of presentation, we will consider the particular experimental conditions relevant to fluorescent decay measurements that use a polarized light source whose temporal structure is independent of the wavelength. Such conditions are met by synchrotron light sources. The relevant modifications to the equations when this condition is relaxed are set out in Appendix I. Furthermore, although the methods presented in this paper are quite general, they can, in principle, be applied to phase-modulation fluorometry, polarized pulse-fluorometry, and energy transfer pulse 
fluorescence. In the present work we restrict ourselves to analysis data from unpolarized pulse fluorescence experiments.

In the following section we discuss the problem of analyzing pulse fluorometric data. We introduce the MEM in Section 3, indicating why MEM is a method that does not introduce extra structure into the spectrum of decay times not warranted by the data. The following section reports the special computational details of applying MEM unpolarized pulse fluorometric data. These two (rather technical) sections can be omitted on a first reading by those whose main interest lies in practical fluorometry. We then apply our technique to both computer simulated and experimental data of fluorescence in Sections 6 and 7.

\section{INTERPRETATION OF PULSE-FLUOROMETRIC DATA}

In polarized pulse-fluorometry, with an exciting light vertically polarized, the experimenter can measure the parallel and perpendicular polarized components of the fluorescence. The signal results from the spectrum of fluorescent decay times and the depolarization of the emitted light (resulting from the Brownian rotational motion of the molecules), all of which convolved with the shape of the excitation flash. If we assume that there is no energy transfer between fluorophores and no contribution of the excited state to the orientational dynamics, then the parallel and perpendicular components $\left(I_{\mid}\right.$and $\left.I_{\perp}\right)$ of the fluorescent intensity as a function of time after the start of the flash $E(t)$ are given by

$$
\begin{array}{r}
I_{1}(t)=\frac{1}{3} E_{\lambda}(t) *\left[\int_{0}^{\infty} \int_{0}^{\infty} \int_{-0.2}^{0.4} \gamma(\tau, \theta, A) e^{-t / \tau}\right. \\
\left.\cdot\left(1+2 A e^{-t / \theta}\right) \mathrm{d} \tau d \theta \mathrm{d} A\right] \\
I_{\perp}(t)=\frac{1}{3} E_{\lambda}(t) *\left[\int_{0}^{\infty} \int_{0}^{\infty} \int_{-0.2}^{0.4} \gamma(\tau, \theta, A) e^{-t / \tau}\right. \\
\left.\cdot\left(1-A e^{-t / \theta}\right) \mathrm{d} \tau \mathrm{d} \theta \mathrm{d} A\right]
\end{array}
$$

Here $E(t)$ is the temporal shape of the excitation pulse, $\tau$ are the decay times of the fluorophores (as modified by their local chemical or physical environments), $\theta$ are the characteristic time constants of their rotational Brownian motions (assuming the fluorophores are rigidly attached to the macromolecules), $A$ is the initial value of the anisotropy at time zero which is in turn related to the angle between the excitation and emission transition moments in the fluorophore (Wahl, 1983), and $\gamma(\tau, \theta, A)$ is proportional to the number of fluorophores with decay time $\tau$ and rotational motion characterized by $\theta$, which start with a polarization ratio $A$. A more general theory of fluorescence decay is given by Szabo (1984).
The symbol * represents a convolution defined by

$$
E_{\lambda} * f=\int_{0}^{t} E_{\lambda}\left(t-t^{\prime}\right) \cdot f\left(t^{\prime}\right) \mathrm{d} t^{\prime},
$$

where $f$ is an arbitrary function and $E_{\lambda}(t)$ is the temporal shape of the exciting flash at the exciting wavelength after convolution with the instrument response function. Since the temporal shape of a synchrotron radiation pulse is strictly independent of wavelength (Benard and Rousseau, 1974) we can measure $E_{\lambda}(t)$ at the emission wavelength and thus eliminate problems arising from a non-uniform spectral response of the photomultiplier. We can thus omit the subscript $\lambda$ from the following formulae. However if this is not the case, then we can use the signal measured under identical spectroscopic conditions from a sample with a known spectrum of decays (usually mono-exponential) to calculate either the instrumental response or the required convolution (Wahl, 1979).

We can analyze the spectrum of decay times independently of the rotational Brownian motion by considering the sum ${ }^{1}$

$$
\begin{array}{rl}
T(t)=I_{1}+2 \beta I_{\perp} & = \\
E & *\left[\int_{0}^{\infty} \int_{0}^{\infty} \int_{-0.2}^{0.4} \gamma(\tau, \theta, A) e^{-t / \tau} \mathrm{d} \tau \mathrm{d} \theta \mathrm{d} A\right],
\end{array}
$$

where $\beta$ corrects for the different responses of the instrumentation to vertical and horizontal polarized light.

The functional form of Eq. 4 is now independent of $\theta$ and $A$. Thus, integrating over $\theta$ and $A$ first and setting

$$
\alpha(\tau)=\int_{0}^{\infty} \int_{-0.2}^{0.4} \gamma(\tau, \theta, A) \mathrm{d} \theta \mathrm{d} A
$$

we get

$$
T(t)=E * \int \alpha(\tau) e^{-t / \tau} \mathrm{d} \tau .
$$

The same function can be measured directly for an unpolarized emission or with a polarizer orientated at $55^{\circ}$ is used on the emission path, but since the error analysis is different, we refer to this direct measurement as $I_{\mathrm{T}}(t)$. In this paper we only consider the simpler task of recovering the distribution of total fluorescent decay times $\alpha(\tau)$. In a subsequent paper we will treat the full problem of recovering $\tau, \theta$, and $A$ simultaneously.

Our problem is to determine the spectrum of decay constants $\alpha(\tau)$ having measured inevitably sampled, noisy, and incomplete representations of the emitted light and flash profile. Formally, $\alpha(\tau)$ is the inverse Laplace transform of the measured light deconvolved by the flash $E(t)$. However, although deconvolution is well conditioned and relatively stable, inverting the Laplace transform is very ill-conditioned (McWhirter and Pike, 1978). As a result small errors in the measurement of the fluorescence curve

\footnotetext{
'We use the symbol $T(t)$ for the total fluorescent light instead of $S(t)$ to avoid confusion with the symbol, $S$, which is used for entropy.
} 
or the flash profile can lead to very large errors in the reconstruction of $\alpha(\tau)$.

We can view this ill-conditioning, which leads to a multiplicity of allowable solutions, in a different way. Consider the set $A$ of all possible shapes of the curves $\alpha(\tau)$ displayed as a rectangle in Fig. 1. We can calculate "mock" data sets from each $\alpha(\tau)$ in turn (using Eq. 6) and test whether it agrees with the noisy data set. All those $\alpha(\tau)$ that agree with the data within the experimental precision are bounded by a dot-dashed line. Some of these spectra, however, contain unphysical features (corresponding to negative concentrations for example). We reject the unphysical $\alpha(\tau)$ by drawing a dashed line. The remaining subset of spectra (shown shaded) we call the feasible set (Gull and Skilling, 1984a). Every member of this set agrees with the data and is physically allowable.

A full specification of the feasible set is the complete answer to the problem. However, it is, for any nontrivial data analysis problem, much too large (strictly infinitely large) to display, comprehend, or use.

\section{MAXIMUM ENTROPY}

Since we are forced to choose one member of the feasible set, we do so directly by maximizing some function $F[\alpha(\tau)]$ of the spectrum. The function $F$ should be chosen to introduce the fewest artifacts into our chosen distribution.

To better understand the principles of MEM we consider a specific and simple example given in its original guise by Gull and Skilling (1984b). This was deliberately chosen for clarity of exposition rather than practical realism.

We are given a solution of two spherical molecules with rotational time constants $\theta_{1}$ and $\theta_{2}$. The two species are present in the ratio of $1 / 3: 2 / 3$. Two fluorophores with decay time constants $\tau_{1}=1 \mathrm{~ns}$ and $\tau_{2}=4 \mathrm{~ns}$ have been reacted with the mixture and are known to have rigidly attached themselves to the spheres. (Thus we assume these reactions only negligibly change the time constants $\theta_{1}, \theta_{2}, \tau_{1}$, and $\tau_{2}$.)

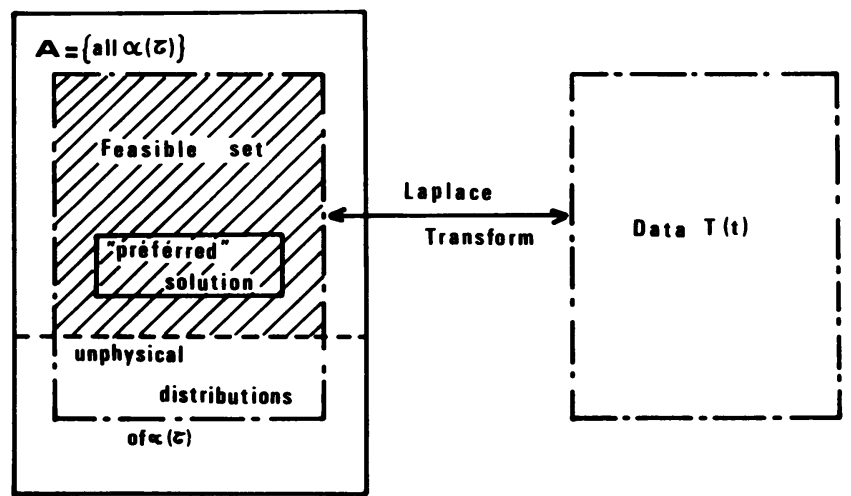

FIGURE 1 Diagram showing the set $A$ of all $\alpha(\tau)$ spectra. (-.-.-) Boundary of the set of $\alpha(\tau)$ which agree with data $T(t)$ within experimental accuracy; (---) boundary of the set of physically allowable $\alpha(\tau)$. The intersection of these two sets is the feasible set (hatched). Inside the feasible set, MEM will choose a "preferred" solution.
We measure the total unpolarized light emitted at time $t_{1}=0.5 \mathrm{~ns}$ and $t_{2}=2 \mathrm{~ns}$ to be $N_{1}=790,508$ and $N_{2}=$ 449,465 counts precisely (without noise) using a light source with an infinitely sharp temporal response at time $t=0$. Our problem is to determine the number of fluorescing centers having fluorescence parameters $\tau_{1}, \theta_{1}, \tau_{2}, \theta_{2}$. That is, we wish to determine the numbers of fluorescent sites $\alpha_{1}\left(\tau_{1}, \theta_{1}\right), \alpha_{2}\left(\tau_{2}, \theta_{1}\right), \alpha_{3}\left(\tau_{1}, \theta_{2}\right), \alpha_{4}\left(\tau_{2}, \theta_{2}\right)$. These two (exact) measurements only give us information about the total amounts of material fluorescing with time constants $\tau_{1}$ and $\tau_{2}$. Using Eqs. 1 and 2, some quick algebra reveals that we had a total of $10^{6}$ fluorescent sites and the two fluorophores are present in the ratio $1 / 3: 2 / 3$.

The feasible set in this example is sufficiently small to display it as a $2 \times 2$ contingency table (Table I). Note that for simplicity of presentation we have normalized the total number of fluorescent sites in Table I (and in Figs. $2 c, 4 a$, $5 c$, and $6 c$ ) to equal one so that our $\alpha$ 's now represent proportions. Thus one-third of the $10^{6}$ fluorescent sites decay with time $\tau_{1}$ and two-thirds with time $\tau_{2}$. Furthermore we also know that one-third of the sites rotate with time constant $\theta_{1}$ and two-thirds rotate with time constant $\theta_{2}$.

We also display three specific choices from this set, namely those with maximal positive and negative correlations and the uncorrelated result. We believe the only rational choice of a single result from such a problem is to choose the uncorrelated result and we should like to choose the function $F[\alpha(\tau, \theta)]$, which gives this result.

It is, of course, very likely that the two fluorophores have different affinities for the two spheres. However, we have no knowledge of whether this will give rise to a positive or negative correlation in our results. We certainly do not

TABLE I

SOLUTIONS TO THE THEORETICAL PROBLEM POSED IN SECTION 3

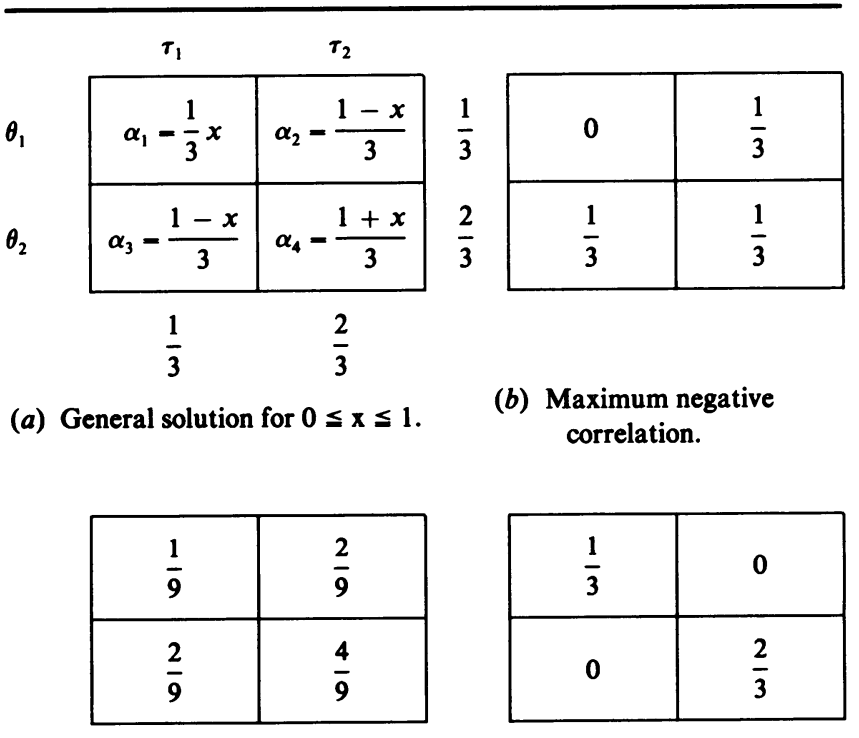

(c) Uncorrelated result.

(d) Maximum positive correlation. 
wish our mathematical optimization function to introduce such correlations arbitrarily and hence predict results about the chemistry of the reaction when the data contained no information about these correlations. All other maximization (or regularization) functions introduce correlations into our solution not demanded by the data. Using such an example, it has been proven by Livesey and Skilling (1985) that only the Shannon-Jaynes entropy $S$ (Jaynes, 1983) will give the uncorrelated solution. This function is defined as

$$
S=-\iint \alpha^{\prime}(\tau, \theta) \log \frac{\alpha^{\prime}(\tau, \theta)}{m^{\prime}(\tau, \theta)} \mathrm{d} \tau \mathrm{d} \theta,
$$

where $\alpha^{\prime}(\tau, \theta)$ is the number of the fluorophores with decay constant $\tau$ and Brownian rotation parameter $\theta$, and $m^{\prime}(\tau, \theta)$ is the model that encodes our prior knowledge about the system before the experiment (see below). A more complete proof has been given by Shore and Johnson (1980). Any other "regularizing function" (Tikhonov and Arsenin, 1977) will impose extra structure in our spectrum without justification. Thus Table II shows the results for some "popularly" used regularization functions. As we have stated, all the functions give either positive or negative correlations with the exception of Shannon-Jaynes entropy. We invite skeptical readers to try maximizing any other function to find their "chosen" solution to this simple problem.

At present, however, we are only concerned with obtaining the shape of the marginal distribution $\alpha(\tau)$, and the entropy reduces to the one-dimensional integral

$$
S=-\int \alpha(\tau) \log \frac{\alpha(\tau)}{m(\tau)} \mathrm{d} \tau,
$$

where $\alpha(\tau)$ is the number of fluorophores that decay with time constant $\tau$.

Although we cannot measure data sets in which different parts of the spectrum decouple completely from the data, we contend that the Shannon-Jaynes entropy will still introduce the least correlations and hence the least spurious structure into our reconstructed distribution of decay times.

Indeed one of the tenets of the axiomatic derivation introduced by Shore and Johnson (1980) and generalized by Livesey and Skilling (1985) is that the coordinate system and order of data analysis should have no effect on the shape of the reconstruction. Whether we marginalize our equations first and compute the one-dimensional distri-

TABLE II

\begin{tabular}{ll}
\hline Regularization function & $p\left(\tau_{1}, \theta_{1}\right)$ Correlations \\
Shannon entropy $-\Sigma p_{i} \log p i$ & $1 / \%=0.111$ Uncorrelated \\
Least squares $-\Sigma p_{i}^{2}$ & $1 / 12=0.0833$ Negative \\
Burg entropy $\Sigma \log p_{i}$ & $(\sqrt{17}-1) / 24=0.13013$ \\
& Positive \\
Intermediate form $\Sigma p_{i}^{1 / 2}$ & 0.12176 Positive \\
\hline
\end{tabular}

bution of $\alpha(\tau)$ or whether we reconstruct the full threedimensional distribution of $\gamma(\tau, \theta, A)$ and then marginalize this solution to obtain $\alpha(\tau)$ will have no effect on the reconstruction. Since we are forced to use the ShannonJaynes entropy in the two-dimensional reconstruction, we must use the Shannon-Jaynes entropy in the one-dimensional reconstruction whether or not the data were measured as separate independent polarized components or at a magic angle, using an unpolarized or a polarized source. Theoretically, at least, the identical data set could be provided as the separate independent polarized components and our conclusions about the distribution of $\alpha(\tau)$ should not depend on the accident of how the data were measured. This will only be true, in general, if we use the same regularization function in the one-dimensional analysis as the two-dimensional analysis above.

However, practical measurements are noisy. It would be wrong to fit the data exactly because the noise would then be interpreted as if it were true signal. We chose to bound the feasible set by a chi-squared statistic.

$$
C=\frac{1}{M} \sum_{t=1}^{M} \frac{\left(I_{t}^{\text {calc }}-I_{t}^{\text {obs }}\right)^{2}}{\sigma_{t}^{2}} \leqq 1.0,
$$

where $M$ is the number of (independent) observations of the fluorescent intensity at times $t$. The variance $\sigma_{t}^{2}$ should take proper account of the inaccuracy of the computation of the calculated intensity arising from uncertainties in the shape of the exciting flash $E$. At values of $C>M$ the calculated and observed data are not in agreement and for $C<M$ we start to fit the noise in $I_{t}^{\text {obs }}$. Note that we do not reduce $M$ by the number of parameters in our spectrum. Indeed these could be greater in number than our observations. We are not using Eq. 9 to find the optimal set of parameters in $\alpha$ space, but instead, we are using it in data space to test whether or not computed data is in satisfactory agreement with our measured data. Other statistical tests could be used to delimit the feasible set, but we found that the above is easy to encode, has powerful statistical arguments justifing its use (Jaynes, 1983) and is sufficiently discriminating for both pulse fluorescence and quasi-elastic light-scattering experiments (Livesey et al., 1986).

The model $m(\tau)$ gives our best a priori guess for the structure of $\alpha(\tau)$. In the above problem we were given (and hence a priori knew) the decay times and the appropriate model (implicitly assumed in the proof) is uniform over $\tau$ space. However, Jaynes (1968, 1983 [p. 114]) showed that if one has no knowledge of the values of the decay constants expected then one must choose

$$
m_{i}=\frac{1}{\tau_{i}} .
$$

A brief resume of his proof is given in Appendix II, which further shows that by transforming variables and working with equally spaced points in $\log \tau$ space the model 
$m_{i}$ becomes constant. In the addition the log space $\tau$ is equivalent to a $\log$ space $\nu(\nu=1 / \tau)$, which is also convenient for spectroscopy kinetics interpretation. If the user has knowledge of correlation in $\alpha(\tau)$ then these can (and should) be introduced by setting up an approximately different model $m_{i}$. Maximum entropy will then return that reconstruction, which provides no more correlations than those present in the data and model.

We note the MEM solution also displays many secondary, generally useful properties. (a) Because of the log term in $S=\Sigma-\alpha \log \alpha / m$, the spectrum $\alpha(\tau)$ is automatically positive. ${ }^{2}(b)$ The spectrum $\alpha(\tau)$ is smooth. (c) It only shows features (in particular the resolution of close peaks) if demanded by the data. $(d)$ Provided the digitization is sufficiently fine to show all the relevant detail, the shape of the spectrum is independent of the number of points used to display it. (e) It is robust to noise, the sense that analyses of the same data that have different random samples of noise added to them are very similar (Livesey et al., 1986) particularly in comparison to a two, three, four, etc., exponential fit. However, if the data change significantly with respect to their estimated accuracy then the reconstruction will be different. $(f)$ For data that are linear functions of the spectrum (this includes the Laplace transform and convolutions), the MEM solution is unique. This is important algorithmically as we can then provide a simple local test (namely that the entropy gradient $\partial S / \partial \alpha_{i}$ and chi-squared gradient $\partial C / \partial \alpha_{i}$ are strictly antiparallel) that our computer program has indeed found that reconstruction that maximizes $S$ subject to the constraint that $C=1$. Similarly, but unlike nonlinear least squares, the final solution is independent of the initial guess of the reconstruction, which we always set to be flat.

Finally we note that, in general, the MEM solution does not have a statistical interpretation. It is no more likely than any other member of the feasible set, but remains the best choice we can make for any given data set. For further discussion see Livesey and Skilling (1985).

\section{PRACTICAL COMPUTATION}

We used the Cambridge maximum entropy suite of subroutines to find that reconstruction of $\alpha(\tau)$, which has the maximal entropy, $S$ (Eq. 8b) subject to the constraint that its calculated data gave a chi-squared value $C$ (Eq. 9) less than or equal to one. A full description of the Cambridge algorithm has been given by Skilling and Bryan (1984). This algorithm provides a single subroutine call (MEMPRM), which will advance an initial spectrum towards the reconstruction which has maximal ShannonJaynes entropy subject to chi-square equalling one. The

\footnotetext{
${ }^{2}$ Difference spectra should be treated by solving for each distribution and then subtracting these results, otherwise the constraint of positivity will be lost in the analysis; see Laue et al. (1986) for an example for NMR of difference spectra reconstructed by MEM, and see Appendix III for a more complete discussion of this property.
}

TABLE III

EFFECT OF THE FLASH SHIFT ON MEM ANALYSIS

\begin{tabular}{ccccc}
\hline & \multicolumn{5}{c}{ MEM } \\
\cline { 2 - 5 } Shift & $\tau_{1}$ & $\tau_{2}$ & $A_{1}{ }^{*}$ & $C$ \\
\hline$p s$ & $n s$ & $n s$ & & \\
-60 & 1.48 & 4.11 & 0.597 & 4.92 \\
-40 & 1.39 & 4.08 & 0.598 & 2.69 \\
-20 & 1.30 & 4.04 & 0.599 & 1.40 \\
-8 & 1.24 & 4.03 & 0.599 & 1.10 \\
0 & 1.20 & 4.00 & 0.600 & 1.00 \\
+8 & 1.22 & 3.99 & 0.599 & 1.01 \\
+20 & 1.23 & 4.02 & 0.596 & 1.03 \\
+40 & 1.26 & 4.02 & 0.590 & 1.10 \\
+60 & 1.28 & 4.03 & 0.583 & 1.22 \\
\hline
\end{tabular}

*The parameter $A_{1}$ corresponds to the relative ratio of the integrated areas under the two peaks centered at $\tau_{1}$ and $\tau_{2}$. It does not take into account the amplitudes of the structure at the short decay times (see Fig. $4 c)$.

user must supply a subroutine to calculate synthetic data from a trial reconstruction of the spectrum. We used recursive algorithm of Wahl (1979), modified for long decay constants to allow for the continuing fluorescence arising from earlier excitations (see Appendix I). These coefficients were calculated once and for all and stored in a $j \times k$ matrix where $j$ is the number of image points (typically 150 or more) and $k$ is the number of data (typically no more than 510). The user must also supply a subroutine to calculate the transpose of this operation.

After setting up the data, accuracies $\left(2 / \sigma_{k}^{2}\right)$ and initial starting map (always flat in $\log \tau$ space) into the appropriate areas used by the suite of subroutine, typically 50-100 calls to MEMPRM were needed to reach the final MEM solution.

MEMPRM is an optimization algorithm specifically designed to maximize the Shannon-Jaynes entropy subject to chi-square constraints on the data. It uses multiple search directions, local quadratic models for both $S$ and $C$, and predictions of the search directions with which to increment $\alpha_{i}$.

Under some experimental conditions, measurements can be "contaminated" by a small amount of the excitation flash being scattered by the solution under study or by a temporal jitter during the flash measurement. To allow for this, we can add an extra point $\alpha_{0}$ to the spectrum to calculate the contribution of a species with a decay constant $\tau=0$. This allows us to remove any contribution to the signal, which has exactly the same shape as the flash. MEM then adjusts the proportion of spectrum at this point to take account of this experimental artifact.

If the decay constants in the sample are considerably shorter than the repeat period between the flashes $(73$ or $36.5 \mathrm{~ns}$ for the Anneau de Collision d'Orsay synchrotron machine in single or double bunch mode) we can obtain a good estimate of the background and its variance by averaging over sufficient measurement channels before the 
flash occurred. However, when the decays are large or these estimates are poor, we must calculate the background more accurately. Once again, an extra point $\alpha_{\infty}$ is added to the distribution. The synthetic data from this point are set to be constant for all $t$ and is not, therefore, strictly a decay with $\tau=\infty$. MEM forces this point to be positive, so we start with a (slight) underestimate of the background and upwards adjust it until the proportion of the distribution in this channel is acceptably low (typically $<10^{-4}$ ). If the background is set too high, then this value becomes very small $\left(<10^{-10}\right)$ in an attempt to go negative, the target value of chi-squared cannot be reached and the residuals are all positive at long measurement times.

The use, by MEM, of the chi-square statistic $C=1(\mathrm{Eq}$. 9) as a hard constraint requires us to provide good estimates of the variances $\sigma_{t}^{2}$. We assumed the counting statistics for each independent measurement were Poisson and used the total number of counts (including background) at each point as our estimate of its variance. The total fluorescence calculated by summing the parallel and perpendicular components (Eq. 4) is not, however, Poisson and its variance is given by

$$
\operatorname{Var}\left(T^{\text {obs }}\right)=\operatorname{Var}\left(I_{1}^{\text {obs }}\right)+4 \beta^{2} \operatorname{Var}\left(I_{\perp}^{\mathrm{abs}}\right),
$$

where $\operatorname{Var}\left(T^{\text {obs }}\right)$ is the variance of $T_{t}$, etc.

The synthetic data contain errors resulting from the inaccuracies in the measurement of the flash. Once again, assuming the measurements of the points making up the flash $E_{t}$ to be Poisson, then their variance is given by the number of counts measured. Differentiating Eq. 3 and using standard error propagation analysis we find the variance in the synthetic data is given by

$$
\operatorname{var}\left(I_{t}^{\text {calc }}\right)=\sum_{k=1}^{t}\left[\frac{E_{t-k+1}}{E_{\text {tot }}}\left(\epsilon E_{t-k+1} Z\right)\right]^{2},
$$

where $E_{\text {tot }}$ is the integral of $E(t), Z$ is the calculated decay law, and $\epsilon=1 / 2$ for $k=1$ and $k=t$, otherwise $\epsilon=1$. The errors in the synthetic and observed data curves are uncorrelated so the variance $\sigma_{t}^{2}$ is

$$
\sigma_{t}^{2}=\operatorname{var}\left(I_{t}^{\text {calc }}\right)+\operatorname{var}\left(I_{t}^{\text {obs }}\right) .
$$

\section{COMPUTER SIMULATIONS}

The computer programs were extensively tested by creating artificial data from a known spectrum of decay times and attempting to recover the spectrum. In all cases the reconstructed spectrum was a very good representation of the original and generally showed more interpretable information than an analysis using constrained nonlinear least squares. We present here a typical test.

The trial spectrum consisted of two delta functions of heights 0.6 and 0.4 units at decay times of 1.2 and $4.0 \mathrm{ns,}$ respectively.

The resulting exponential decay was convolved with an experimental flash profile measured at the pulse-fluores- cence station at LURE (FWHM $=1.6 \mathrm{~ns})$. The "data" were produced at 430 points each separated by $0.080 \mathrm{~ns}$. Synthetic noise drawn from a Gaussian probability distribution of zero mean and variance equal to the "fluorescence" counts was added to each point.

The experimental flash profile and the synthesized data are shown in Fig. $2 a$. The spectrum was reconstructed on 100 points equally spaced in $\log \tau$ between 0.1 and $10 \mathrm{~ns}$ and is displayed in Fig. $2 c$. Two major, tolerably symmetrical, peaks are visible corresponding to the original two delta functions with their "centroids" at 1.2 and 3.99 ns. The proportional integrated areas under the two peaks are 0.599 and 0.401 in close agreement with the originals. There are no significant evidence for any asymmetry in the peaks not for any other structure. Other tests have shown that if the allowed range of decays is too small than a peak is seen to pile-up at one end of the range needing extended. There is no evidence for this in this reconstruction, showing that the reconstruction range was adequate.

Chi-square reached 1.00 (indeed this is necessary for the maximum entropy technique), showing we were truly inside the feasible set. As expected from this chi-square value and as shown in Fig. $2 a$ the data calculated from the reconstructed spectrum fit the simulated data very well and the residuals (Eq. 9) appear random (Fig. $2 b$ ).

We analyzed the same data set using constrained nonlinear least squares method. Not surprisingly, least-squares find strong evidence for two delta functions close to the originals, and can fit the data with a chi-squared equal to 1.00 .

MEM can resolve close pair of exponentials with a ratio of lifetimes of 1.4 as illustrated in Fig. 3. The trial consisted of two delta functions of height 0.6 and 0.4 units with lifetimes of 5.0 and $7.0 \mathrm{~ns}$, respectively. The "data" were produced at 440 points each separated by $0.10 \mathrm{~ns}$. Synthetic noise was added as above. The resolution power is obviously dependent on the data points, the sharpness of the excitation, and the number of data points (Brochon, J. C., and A. K. Livesey, manuscript in preparation).

However MEM can also analyze broad input distributions (or mixtures of broad and sharp features), which are poorly reproduced by constrained least squares analysis by a few exponentials. Examples of such reconstructions from Laplace transform data can be found in Livesey et al. (1986 and 1987).

\section{SCATTERED LIGHT AND LAMP PROFILE SHIFT}

To balance the scattered light or the color effect occurring in the fast photomultiplier tubes (Wahl et al., 1974; Heisel et al., 1979a), some experimenters introduce an inaccurately determined shift to the lamp profile measured at the excitation wavelength (Imhoff and Birch, 1982). Although the very nonlinear behavior of maximum entropy allows many unmeasured experimental parameters to be determined automatically and hence corrected (Sibisi, 1983b; 

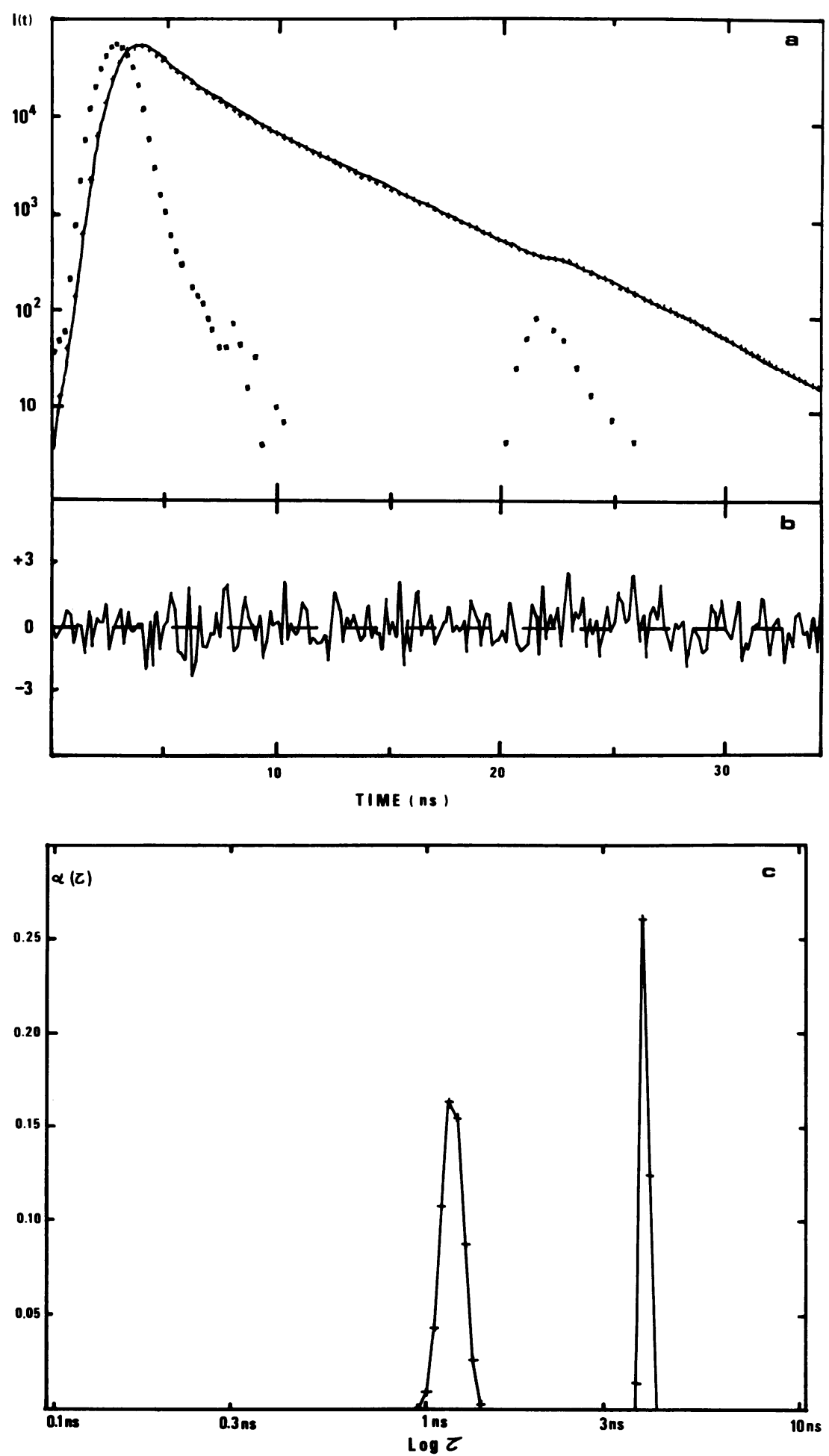

FIGURE 2 (a) The experimental flash profile $(\square)$, the synthetized data $(+)$, and the calculated curve $(-)$ are represented in semilogarithmic scale. The "data" were produced at 430 points each separated by 80 ps. (b) Plot of the weighted residuals. (c) MEM reconstructed spectrum of $\alpha(\tau)$ on 100 points equally spaced in $\log \tau$ between 0.1 and $10 \mathrm{~ns}$. The two peaks are centered at 1.2 and 3.99 ns. The $\alpha(\tau)$ have been normalized and now represent proportions. The total "counts" of noisy data were $\approx 2.9 \times 10^{6}$ and the value of the top channel was 53,948 .
Scott, 1981), we were unable to use the same autocalibration technique to determine the shift of the lamp profile, which is a major source of error in practical pulsefluorescence analysis. However, we can use the above example to test the effect of varying this parameter on the maximum entropy reconstructions.

We thus attempted to reconstruct the data set depicted in Fig. $2 a$ with the lamp profile shifted from its true position. A simple three points interpolation was used to determine the values at intermediate points. We call the shift negative if the lamp profile used for the recovery is early with respect to the lamp profile, which produced the fluorescent decay data. If the shift is negative the "data" can only be fitted with a spectrum contraining negative peaks. Since we postulated a positive spectrum of exponential terms, entropy forbids such negative regions and, as a 


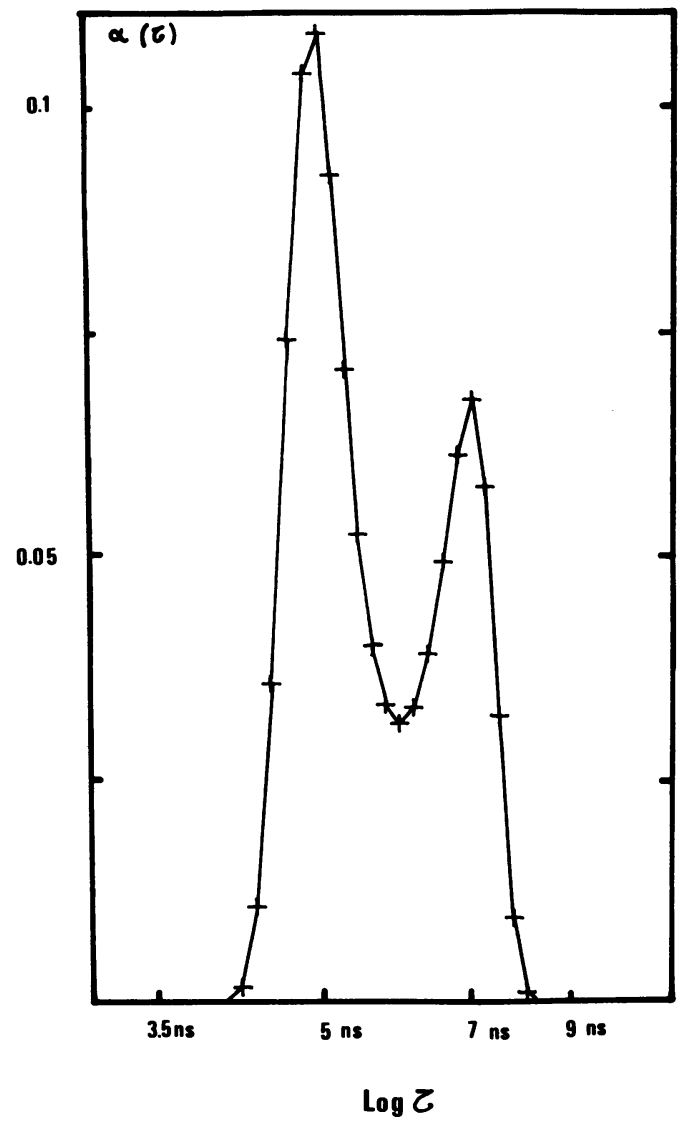

FIGURE 3 MEM reconstructed spectrum of $\alpha(\tau)$ on 30 points equally spaced in $\log \tau$ between 3.5 and $9.0 \mathrm{~ns}$. The two peaks are centered at 4.94 and $7.09 \mathrm{~ns}$. The total "counts" of noisy data were $\simeq 58 \cdot 10^{6}$ and the value of the top channel was $8 \cdot 10^{5}$.

result, chi-squared cannot fall to 1.0 . We found that the spectrum could not be fitted with a shift of $-0.02 \mathrm{~ns}$ $(-0.25$ channel $)$. At $-0.008 \mathrm{~ns}(-0.1$ channel) a chisquared of 1.1 can be reached, but the reconstruction is impressively similar (Fig. $4 a$ ) to the reconstruction found with zero shift.

Close inspection of the residuals (Fig. $4 \mathrm{~b}$ ), however, shows that even this small shift, which has little effect on the reconstruction, can be detected as causing a small nonrandom asymmetrical peak in the distribution of the residuals spanning the maximum of the lamp-pulse.

Positive shifts can be partially compensated for by creating pulse structure at short decay times. However we found that a shift of +0.04 ns $(0.5$ channel $)$ prevented chi-squared reaching 1.0 and the spectrum shows a small structure at low decay time (Fig. 4 c). This structure always piles up at the low decay time boundary; however, the boundary is set low and can thus be partially distinguished from true structure with decay times shorter than the allowed range. Once again positive shift can be recognized in the pattern of residuals (Fig. $4 c$ ) as giving the opposite asymmetric peak to that observed with a negative shift. A positive shift of $0.008 \mathrm{~ns}(0.1 \mathrm{channel})$ could not be distinguished by its effect on the residuals but produced
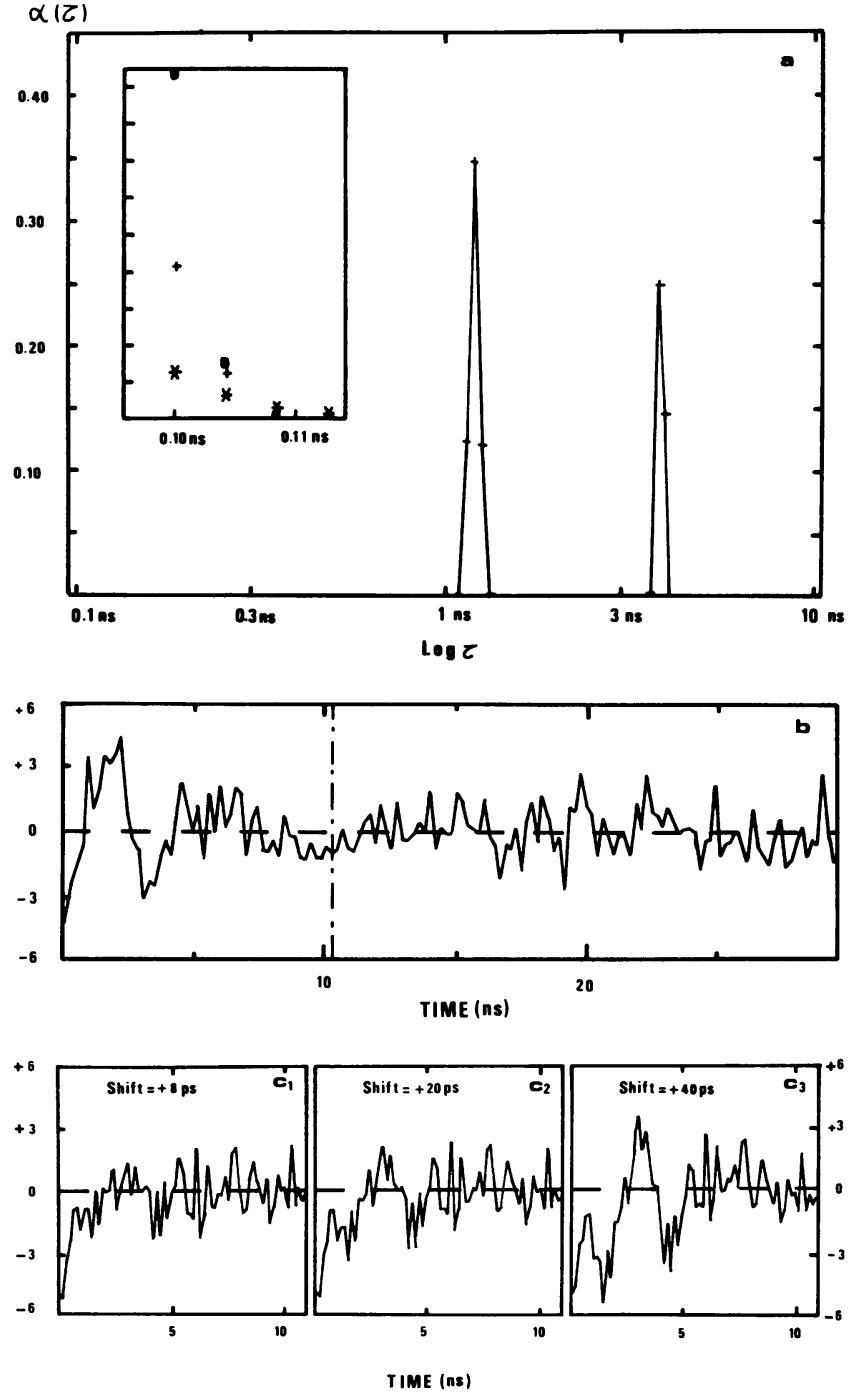

FIGURE 4 Synthesized noisy data analysis: (a) MEM reconstructed spectrum of $\alpha(\tau)$ on 100 points equally spaced in $\log \tau$ between 0.1 and 10 ns for a flash profile shift of $\mathbf{- 8} \mathrm{ps}$. In the upper left window the structure in $\alpha(\tau)$ spectra at short decay times is displayed for positive shifts: $\left({ }^{*}\right)$ $8 \mathrm{ps}+; 20 \mathrm{ps}$; (.) $40 \mathrm{ps}$. (b) Plot of the weighted residuals corresponding to the analysis with a negative 8-ps shifted flash. (c) Plot of the first third of the weights residuals obtained with positive shifted flash of 8,20 , and $40 \mathrm{ps}$, respectively.

correspondingly smaller artificial structure at low decay times without affecting the structure of the decay spectrum at longer time scales.

Experimentally we always measure the excitation pulseshape before and after a fluorescence experiment and if any significant shifts are observed, the experiment is rejected. Chi-squared and a visual inspection of residuals then provide powerful tests for further eliminating data with any remaining small shifts. Our experience suggests the remaining small range of allowable shifts will have little or no effect on the reconstructions at time constants greater than about five times the experimental channel width. If the user is interested in decay structure at shorter times then a series of spectra (say three or five) should be 


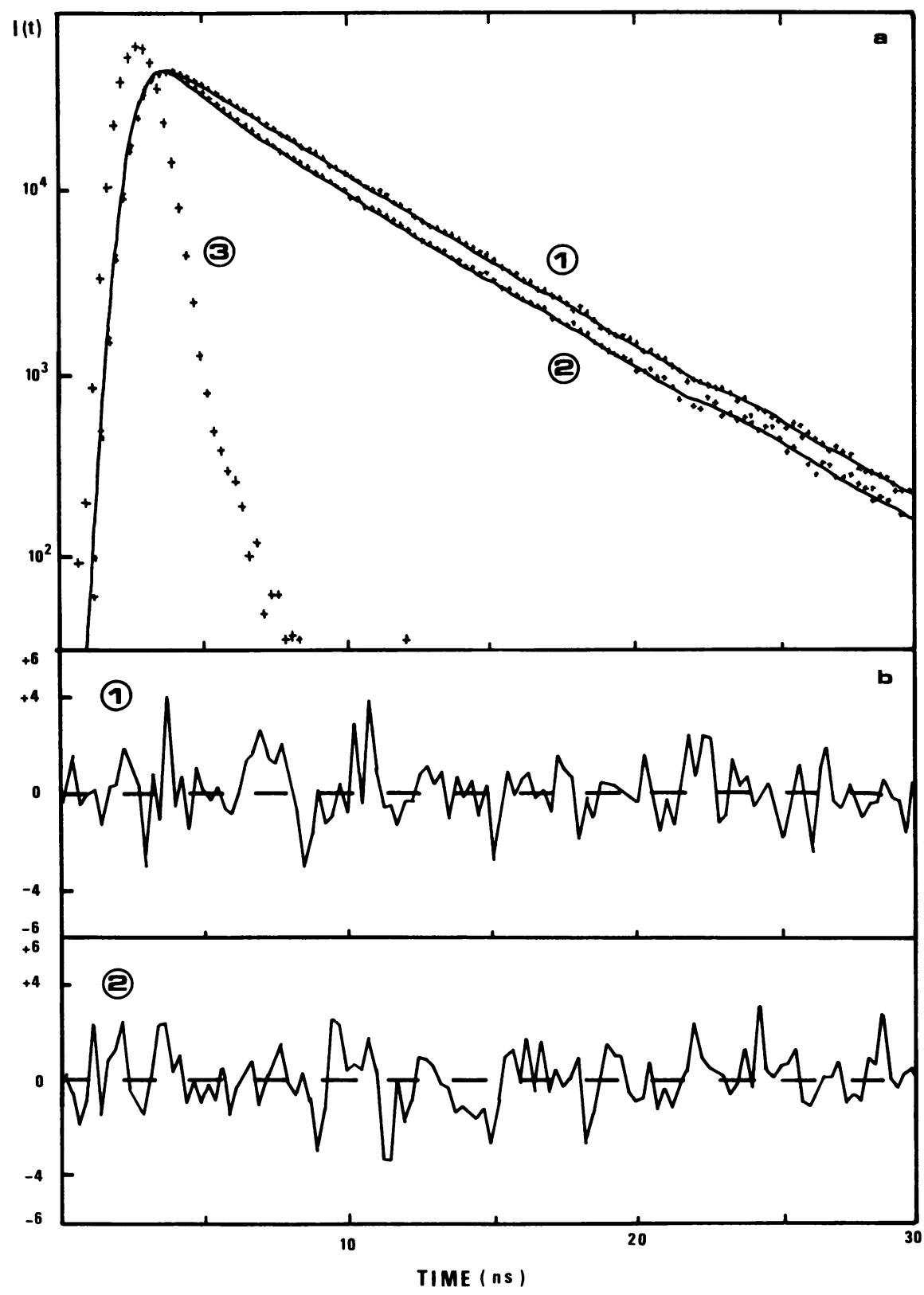

FIGURE 5 (a) Fluorescence decay curves of L-trytophan at two emission wavelengths $(\Delta \lambda=4 \mathrm{~nm})$ : (1) $390 \mathrm{~nm}$; (2) $320 \mathrm{~nm}$. +++ , experimental curves; - calculated curves. (3) The experimental flash profile. The excitation wavelength was $280 \mathrm{~nm}(\Delta \mathrm{A}=4 \mathrm{~nm})$ and temperature $10^{\circ} \mathrm{C}$. (b) Plot of the weighted residuals corresponding to two curves ( $I$ and 2$)$. (c) MEM reconstructed spectra of $\alpha(\tau)$ on 100 points equally spaced in $\log \tau$ between 0.08 and $8 \mathrm{~ns}$ for the two emission wavelengths $390 \mathrm{~nm}$ $(-)$ and $320 \mathrm{~nm}(+)$. The spectrum of $\alpha(\tau)$ for 390-nm emission is plotted in arbitrary units to normalize peak maxima for longer decay times.

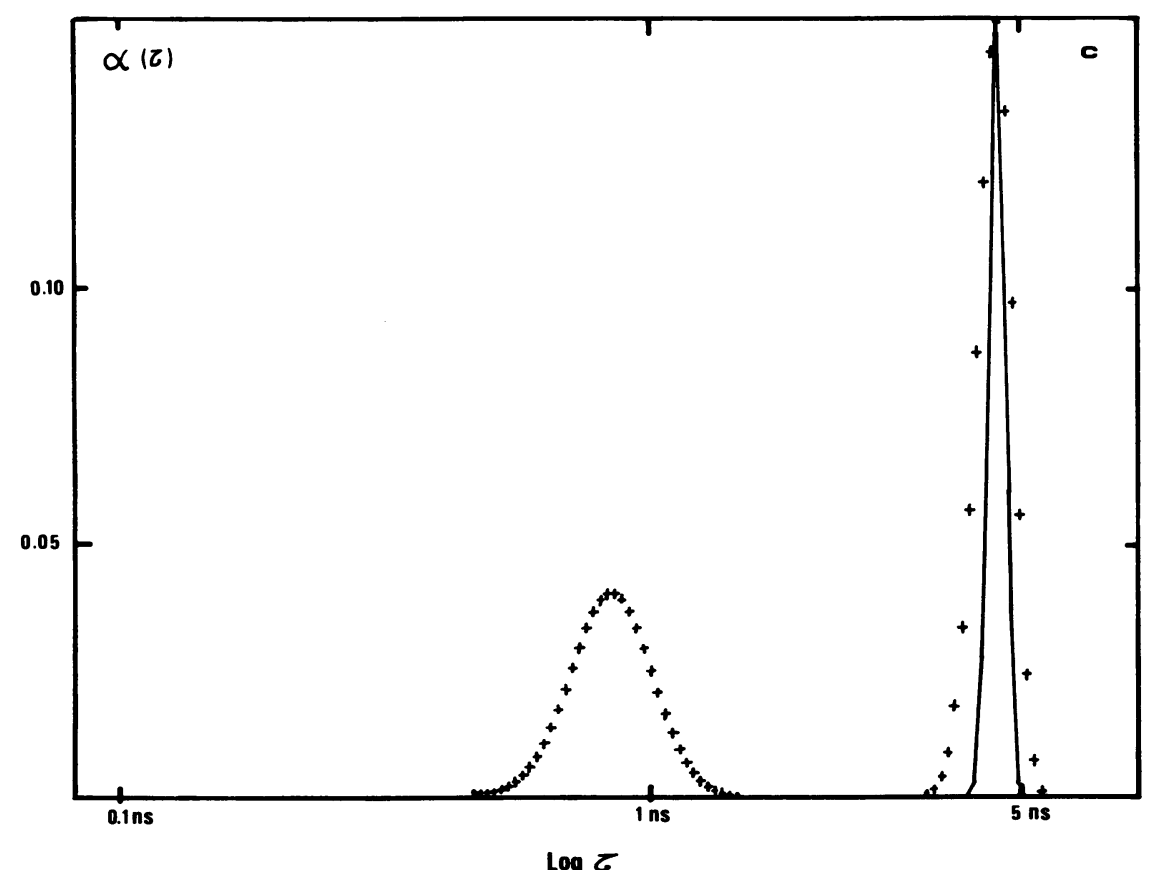


calculated with shifts spanning the allowable range, and all those spectra that are significantly different should be displayed as the results of the experiment.

\section{FLUORESCENCE DECAY MEASUREMENTS}

Synchrotron radiation emitted by the electron storage ring ACO was used as the excitation light pulse. The repetition rate was $13.6 \mathrm{MHz}$. Fluorescence decays were measured by the time correlated single photon counting technique (Ware, 1971; Yguerabide, 1972; Wahl, 1975) and the experimental set-up has been previously described (Jameson and Alpert, 1979; Brochon, 1980; Privat et al., 1985).

\section{MATERIALS}

All solvents were freshly distilled at least twice and were spectroscopically pure.

L-Trytophan, from Sigma Chemical Co. (St. Louis, MO), was recrystallized from ethanol/water 80:20 ( $\mathrm{vol} / \mathrm{vol}$ ). The compound appeared to be homogeneous by thin layer chromatography on silica-gel plate (Merck, Darmstadt, FRG) in $n$-butanol/acetic acid/water 60:20:20 when it was revealed either by ninhydrin or by fluorescence. This sample was dissolved in $0.01 \mathrm{M}$ acetate buffer adjusted to $\mathrm{pH}$ 5.4.

Trans-stilbene from Sigma Chemical Co. was used without any further purification in cyclohexane.

\section{RESULTS}

\section{L-Tryptophan}

The measured data points from L-tryptophan together with the (wavelength independent) excitation pulse are shown in Fig. $5 a$ with a logarithmic ordinate. On this plot the decay at $390 \mathrm{~nm}$ appears to be a straight line, suggesting that the decay could be fitted by a straight line whose slope would give the appropriate decay constant. The decay at $320 \mathrm{~nm}$ is, however, more complicated involving at least two significant time constants. The corresponding MEM reconstructions of the distributions of decay constants are presented in Fig. $5 c$, and the dotted lines on Fig. $5 a$ show the fit to the data by these spectra. At $390 \mathrm{~nm}$ there is only evidence for one strong peak centered at $4.46 \mathrm{~ns}$ with half-width of $\sim 0.1 \mathrm{~ns}$. At $320 \mathrm{~nm}$ the spectrum of decay constants has two peaks centered at 0.82 and $4.35 \mathrm{~ns}$. The relative integrated areas under these peaks are 0.623 and 0.377 , respectively. These results are in good agreement with several previously published results (Szabo and Rayner, 1980; Robbins et al., 1980; Privat et al., 1985). It is worth emphasizing the excellence of the fit to the data. Provided a large enough range of decay values is fitted with sufficiently closely spaced points, chi-squared must come down to 1.0. Any attempt to fit a chi-squared below this value leads to the danger of reproducing noise in the data as unwarranted false structure in the reconstruction. Similarly, if we cannot attain a chi-squared of 1.0 there is probably a mistake in the experiment or its analysis. Any "piling up" of the spectrum against the edges of the allowed time domain suggests the time domain is too restricted. Re-running the analysis with a larger time domain will bring down the value of chi-squared. Otherwise a display of the residuals will often reveal either an individual abberant point or a systematic trend such as the pickup of a radiofrequency field. The relevant data can be discarded or the experimental procedure modified accordingly. In this case, however, the residuals appeared sufficiently random (Fig. $5 b$ ) to have confidence in our result. Note further though that the peak of the shorter time decay is considerably wider than the longer decay. Simulations using delta peaks at 0.82 and $4.35 \mathrm{~ns}$, and similar signal-to-noise ratios, give equally narrow reconstructions to the two peaks, suggesting there is very little evidence for the shorter decay component being a single delta function. Nevertheless fitting these data with two delta functions gives a chi-squared of 1.48 , which is only just rejected by the experiment so that the intrinsic width of this shorter decay peak could well be narrow. Better data will be needed to fully resolve these models.

\section{Trans-Stilbene}

The measured total fluorescent decay and flash profile for trans-stilbene is presented in Fig. $6 a$. The MEM reconstruction of the decay constants, calculated with 150 points equally spaced in $\log \tau$ intervals between 0.005 and $4.0 \mathrm{~ns}$, is shown on Fig. $6 c$. Once again the spectrum can be described by two peaks, centered at 0.075 and $1.5 \mathrm{~ns}$. The first peak strongly dominates the fluorescence. It is interesting to note that such a short decay time can be accurately determined with an excitation pulse of $\sim 1.6 \mathrm{~ns}$ full width half maximum (FWHM). Indeed the convolution spreads the information about the lifetime over many more measurement channels, and since deconvolution is a much better conditioned problem than inverting the Laplace transform, there is a resultant increase in the accuracy of the determination of this short-lived component. Naturally there is a limit to this process. At very short lifetimes $(\sim 0.005 \mathrm{~ns})$ the signal would have died away before the first measurement and the signal will be indistinguishable from scattered flash light. These reconstruction points start to disconnect from the data and MEM will set them equal to the value set in the model.

We found the shape of the spectrum remained unchanged whether or not we included a flash channel (i.e., $\tau_{1}=0$ ). This demonstrated that our experiment was free from any shift or jitter of the flash position, which is critically important to measure such a short decay time.

The peak at $1.5 \mathrm{~ns}$ is very weak, containing only $\sim 0.5 \%$ of the total contents of the spectrum and can be attributed to a residual impurity (Heisel et al., 1979b). Although the temporal resolution with the Laplace transform is poor, the dynamic range of the reconstruction can be very large and any proposed algorithm must be capable of handling such a large range. 

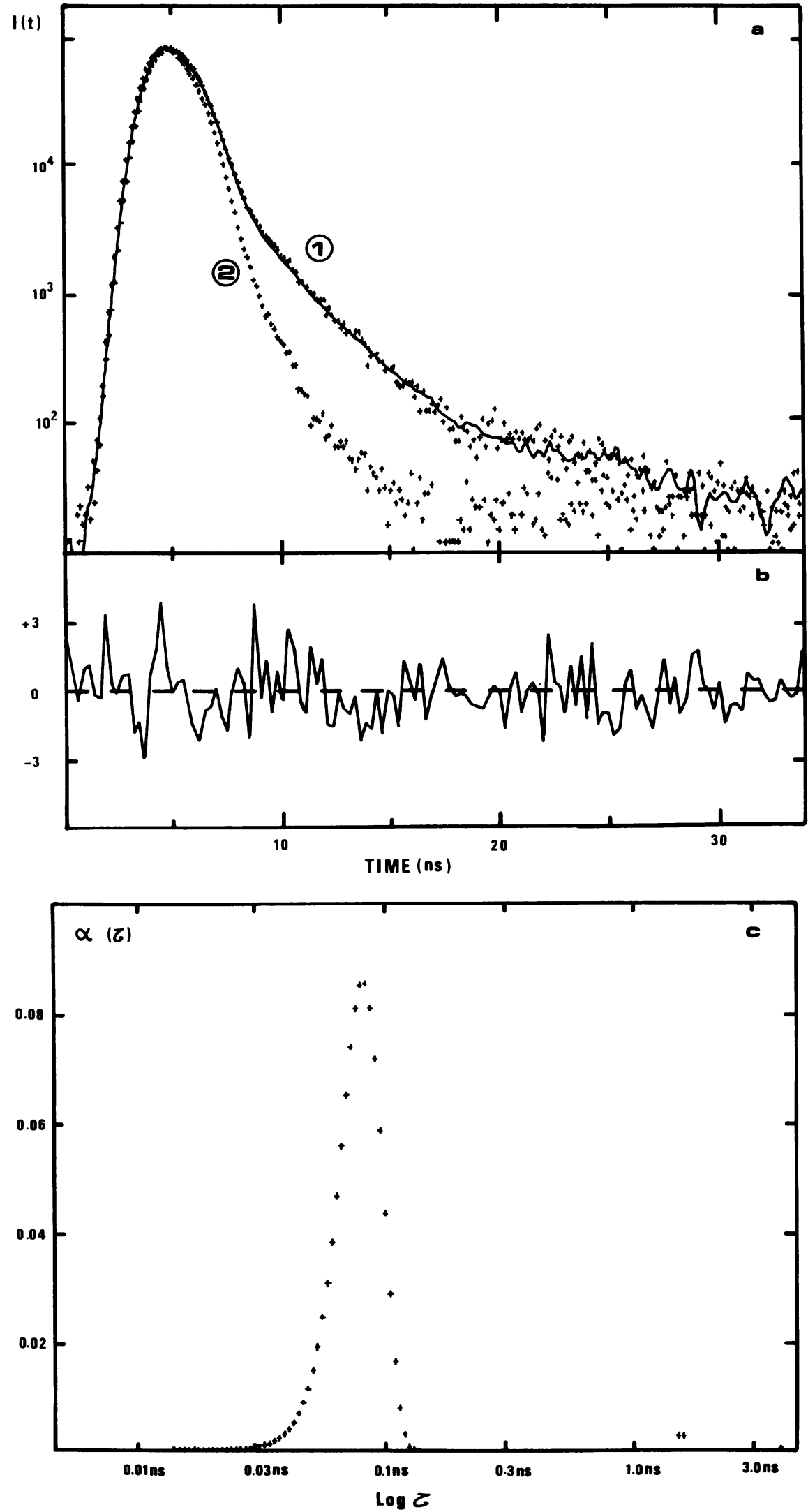

FIGURE 6 (a) Fluorescence decay of transstilbene. The excitation and emission wavelengths were, respectively, $270 \mathrm{~nm}(\Delta \lambda-4 \mathrm{~nm})$ and $370 \mathrm{~nm}(\Delta \lambda=4 \mathrm{~nm})$ and the spectrum was measured at $20^{\circ} \mathrm{C}$. (1) +++ , experimental curve; - calculated curve. (2) The experimental flash profile normalized to the top of fluorescence curve to display the good separation of the two curves, both in the leading and trailing edge. (b) Plot of the weighted residuals. (c) MEM reconstructed spectrum of $\alpha(\tau)$ on 150 points equally spaced in $\log \tau$ between 0.005 and $4.0 \mathrm{~ns}$. There is no structure at very short decay times, indicating the absence of any flash shift during measurements. 


\section{Computation Time}

The analysis of a typical decay curve containing 440 data points and displaying it using $150 \tau$ values takes $\sim 15 \mathrm{~min}$ on a VAX 780. However, a new version of the MEMSYS code reduces this figure by approximately a factor of four (Skilling, J., personal communication). Although we often use two or exceptionally three runs to determine the appropriate range of $\tau$ values or to determine the background levels, the analysis needs no further user intervention, so that all the analysis can run in batch mode overnight. Similarly, since the MEM solution is unique, the results are independent of the starting guess. Indeed we always start from a flat solution to avoid any charge of bias. For comparison, it takes on average $\sim 30 \mathrm{~min}$ to measure both the flashes and fluorescences at the ACO storage ring at LURE.

\section{DISCUSSION}

From the definition of the feasible set, the MEM solution is always an excellent fit to the data. At the given noise level, it neither overfits nor underfits the data. We chose to bound the feasible set by the chi-squared function. This is easy to use, has considerable theoretical justification (Jaynes, 1983), and we have found it sufficiently powerful to analyze Laplace transform data in both pulse fluorescence and quasi-elastic light scattering (Livesey et al., 1986). However, other more sophisticated tests could be used if desired or needed (Bryan and Skilling, 1980). Within this delimited feasible set, MEM chooses that unique solution that has the minimum cross-correlation artifacts.

No model of the parameter distribution is needed beyond the expected uniform distribution in $\log \tau$, which arises from simple consistency arguments (Appendix II). However, if at any stage the user can reject the MEM solution, then he is making use of some prior knowledge of distributions expected. This knowledge should be encoded into the model or measure $m_{\tau}$ (Eq. 10) which will, in turn, improve the reconstruction.

We also find MEM a compelling choice experimentally. It is robust to noise and can handle both sharp and broad distributions of decay times without modification. If the experimental conditions require it, MEM can determine the background level and amount of parasitically scattered radiation. However, we have also found that chi-squared and a visual check of the randomness of the residuals is a powerful test to remove unwanted, nonrandom errors in the data. In particular the radiofrequency shielding of cables and amplifiers at LURE have been considerable enhanced, to ensure that the noise only arises from Poisson counting statistics. The programs need only minimal user intervention and could be run on microcomputers (e.g., PDP 11/60; Burch et al. [1983]), so it is feasible to analyze the previous set of data while an experiment is running to test the quality of data or to find the optimal conditions (temperature, $\mathrm{pH}$, dilution, etc.) for measurement. Finally we note that MEM is completely general and can be applied to other experimental conditions where the fluorescence is described by, for example, the difference of exponential decays or $\exp (-A t-B \sqrt{t})$ etc. Only the algorithm for calculation the convolution (Eq. 6) has to be modified.

\section{APPENDIX I \\ Convolution of an Experimental Decay over a Limited Time Domain}

We consider fluorescent species in solution without interactions, which are characterized by several decay times. The fluorescence intensity per second after an infinitely short excitation is (Wahl, 1975)

$$
F(t)=\sum_{i} \frac{\left[C_{0, i}\right]}{\tau_{0, i}} e^{-t / \tau_{i}},
$$

where $\left[C_{0, i}\right]$ is the initial concentration of excited molecule $i$ at time zero and $\tau_{0, j}$ is the fluorescence decay obtained without any nonradiative process.

In dilute solution this relation becomes

$$
F(t)=\sum_{i} \frac{\epsilon_{i}\left[C_{i}\right] Q_{i}}{\tau_{i}} e^{-t / \tau_{i}}
$$

where $\epsilon_{i}$ is the molar absorbance, $\left[C_{i}\right]$ the molar concentration, and $Q_{i}$ the quantum yield.

Then the general form of the fluorescence decay is

$$
F(t)=\sum_{i} \frac{I_{i}}{\tau_{i}} e^{-t / \tau_{i}}
$$

with an excitation having a definite time profile $E(t)$ we have

$$
I_{0}(t)=E(t) * F(t) .
$$

With synchrotron radiation $E(t)$ is measured correctly at the fluorescence wavelength, otherwise the convolution is obtained from a reference compound as previously described (Wahl, 1974, 1979; Zuker et al., 1985).

The time interval between two excitation pulses is $\sim 73.6 \mathrm{~ns}$. The measured fluorescence decay of a sample having longer decay times corresponds to the superposition of the responses of several previous pulses. With synchrotron radiation there is strictly no excitation between pulses. So a simple extrapolation of the fluorescence decay contribution from a previous flash is given by the following relation:

$$
I_{k}(t)=I(N) \exp -\left[\frac{(k-1)}{\mathrm{T}_{0}}\right] \exp \frac{-t}{\tau},
$$

where $I(N)$ is the last calculated value of the convolution $I_{0}(t)$ in Eq. A1.4, $T_{0}$ corresponds to the time interval between flashes $(73.6$ or $36.8 \mathrm{~ns}$ in single or double bunch mode at ACO), and $k$ is the order of the previous flash taken in account. The computation is stopped when $I_{k}(t)$ reached a very low value, typically $\sim 10^{-5}$.

The convolution is the sum of the contribution of different excitation pulses:

$$
I(t)=I_{0}(t)+\sum_{k=1}^{P} I_{k}(t)
$$




\section{APPENDIX II}

\section{Calculation of an A Priori Model for Multiexponential Decays}

Before we can use the Shannon-Jaynes entropy (Eq. 8b) we must define our prior knowledge and encode it into the model $m(\tau)$. We start from the objective statement that "in two problems where we have the same prior information, we should assign the same prior distribution."

We observe data $I(t)$

$$
I(t)=E^{*} \int \alpha(\tau) e^{-t / \tau} \mathrm{d} \tau,
$$

where the $\tau$ are positive scale parameters describing the rate of decay.

Although we know the $\tau$ to be scale parameters, we do not, a priori, know which values will describe the decay of our system. A change of scale should not, therefore, change our conclusions. Two observers measuring the data on two different scales, $t$ and $t^{\prime}=q t$, have their constants related by

$$
\begin{aligned}
t^{\prime} / \tau^{\prime} & =t / \tau \\
\tau^{\prime} & =q \tau .
\end{aligned}
$$

They assign prior distributions $f(\tau) \mathrm{d} \tau$ and $e\left(\tau^{\prime}\right) \mathrm{d} \tau^{\prime}$, respectively. But the value of their functions at any point must be the same; $f(\tau) \mathrm{d} \tau=e\left(\tau^{\prime}\right) \mathrm{d} \tau^{\prime}$ or $f(\tau)=q e\left(\tau^{\prime}\right)$. However, if both observers were equally ignorant before the experiment then they must have been in the same state of knowledge. Thus, their functions $f$ and $e$ must be one and the same $f(\tau)=e(\tau)$.

Combining these equations gives

$$
f(\tau)=q f(q \tau)
$$

or

$$
f(\tau) \mathrm{d} \tau=m(\tau) \alpha \tau^{-1} \mathrm{~d} \tau
$$

Transforming coordinates to $\log \tau$ coordinates gives a useful simplification

$$
\begin{gathered}
r=\log \tau \\
\mathrm{d} r=\frac{1}{\tau} \mathrm{d} \tau .
\end{gathered}
$$

Thus the measure is constant in $\log \tau$ space and it is for this mathematical convenience that we chose to work at equally spaced intervals in $\log \tau$ space.

\section{APPENDIX III}

\section{Application of the Positivity Constraint in Pulse-Fluorescence}

The constraint of positivity is not imposed as an axiom necessary to derive the maximum entropy formalism. Confusion over this principle has arisen in the past because the Shannon-Jaynes algorithm has been derived for the very restricted case that the resulting spectrum is a distribution of probabilities and hence must have a value lying between 0 and 1 such that the total sum of probabilities is 1 . However, this is unnecessarily restrictive and inapplicable for pulse-fluorescence; instead we turn to the more general variational (as opposed to probabilistic) derivation.

Four axioms are imposed: $(a)$ The variational function $F[\alpha(\tau)]$ should be independent of the type of data being analyzed. $(b)$ The shape of the spectrum should be independent of the units used to display $\alpha(\tau)$.

(c) The shape of the spectrum $\alpha_{1}(\tau)$ to $\alpha_{n}(\tau)$ remains unchanged whether data only pertaining to spectrum points $\alpha_{n+1}(\tau)$ to $\alpha_{m}(\tau)$ is included or not. (d) Given marginal data (such as the example in Table I) we wish to recover the uncorrelated solution.

Surprisingly, perhaps, these four rather general axions are sufficient to uniquely define the variational function $F[\alpha(\tau)]$ and to impose the constraint of positivity. This proof is given in Section 3 of Livesey and Skilling (1985). Shore and Johnson (1980) originally developed this argument but only consider probability distributions. In fact this is unnecessarily restrictive as they do not need to use any of the special properties of probability distributions. Furthermore, Livesey and Skilling (1985) (Sections 4 and 5) show that the statistical model can be misleading and can break down in practice.

Time-resolved fluorescence involves sometimes complicating effects that cause a derivation from simple kinetics law (for an overview see Ware, 1983). For example in the case of excited-state reactions or excitation energy transfers, a fluorescence kinetics can be described as a difference of exponentials leading to a "delayed" rise time of the emission. The negative preexponential term reflects the build-up of corresponding emitting centers from zero concentration at time zero. We can then ask, what positive distribution of centers with a negative amplitude together with what positive distribution of centers with a positive amplitude fit our data such that we introduce the least correlation between these lifetimes. This approach has been successfully applied to NMR where some peaks scatter $180^{\circ}$ out of phase with respect to the others (Laue et al., 1986).

For clarity these authors chose to display their result as a difference spectrum rather than displaying the two separate distributions. A similar problem arose in extended $x$-ray absorption fine structure (EXAFS) where (isolated) atoms of different elements scatter with different phases with respect to each other; in particular, nickel and zirconium scatter almost exactly $180^{\circ}$ differently. However, here it was felt to be more appropriate to display the distribution of both nickel and zirconium (Livesey, 1984). We are currently investigating the applicability of these ideas to the "considerably" worse conditioned problem of pulse-fluorescence data.

We are indebted to the technical staff at LURE for running the machines during beam-time sessions, to the informal maximum entropy group at Cambridge for allowing us to use the Cambridge algorithm and to Fabienne MEROLA for introducing us. J. P. Privat kindly supplied the purified L-tryptophan.

A. K. Livesey gratefully acknowledges the receipt of a European Molecular Biology Organization long term fellowship to work at LURE and thanks G. Bricogne for the invitation to work there. J. C. Brochon thanks Centre National de la Recherche Scientifique for continued financial support of this project.

Received for publication 22 July 1986 and in final form 29 June 1987.

\section{REFERENCES}

Benard, C., and M. Rousseau. 1974. Statistical properties of synchrotron radiation. J. Opt. Soc. Am. 64:1433-1444.

Brochon, J. C. 1980. Protein Dynamics and Energy Transduction. Shin'Ichi Ishiwata, editor. Taniguchi Foundation, Waseda University, Tokyo. 163-189.

Bryan, R. K., and J. Skilling. 1980. Deconvolution by maximum entropy, as illustrated by application to the jet of M87. Mon. Not. R. Astr. Soc. 191:69-79.

Burch, S. F., S. Gull, and J. Skilling. 1983. Image restoration by a powerful maximum entropy method. Comp. Vision Graphics Image Processing. 23:1 18-128.

Gull, S. F., and J. Skilling. 1984a. Maximum entropy method in image processing. I.E.E.E. (Inst. Electr. Electron. Eng.) Proc. 131F:646661.

Gull, S. F., and J. Skilling. 1984b. The maximum entropy method. In Indirect Imaging. J. A. Roberts, editor. Cambridge University Press, Cambridge. 267-279. 
Heisel, F., J. A. Miehe, and B. Sipp. 1979a. Detection et analyse de phenomenes lumineux brefs. Ann. Physique Fr. 4:331-370.

Heisel, F., J. A. Miehe, and B. Sipp. 1979b. Picosecond analysis of trans-stilbene fluorescence. Chem. Phys. Lett. 61:115-118.

Ihmof, R. E., and D. J. S. Birch. 1982. Deconvolution and Reconvolution of Analytical Signals. Application to Fluorescence Spectroscopy. M. Bouchy, editor. Ecole Nationale Supérieure des Industries ChimiquesInstitut National Polytechnique de Lorraine, Nancy, France. 411423.

Jameson, D. M., and B. Alpert. 1979. Synchrotron Radiation Applied to Biophysical and Biochemical Research. A. Castellani and I. F. Garcia, editors. Plenum Publishing Corp., New York. 183-201.

Jaynes, E. T. 1968. Prior probabilities. I.E.E.E. (Inst. Electr. Electron. Eng.) Trans. Biomed. Eng. 4:227-241.

Jaynes, E. T. 1983. Papers on probability, statistics and statistical physics. R. D. Rosenkrantz, editor. D. Reidel, Dordrecht, The Netherlands.

Laue, E. D., J. Skilling, and J. Staunton. 1986. Maximum entropy reconstruction of spectra containing antiphase peaks. J. Magn. Res. 63:418-424.

Livesey, A. K. 1984. The local structure of amorphous binary metallic alloys. Ph. D. Thesis, University of Cambridge, UK.

Livesey, A. K., and J. Skilling. 1985. Maximum entropy theory. Acta Crystallogr. Sect. B. Struct. Crystallogr. Cryst. Chem. A41:113-122.

Livesey, A. K., P. Licinio, and M. Delaye. 1986. Maximum entropy analysis of quasi-elastic Light scattering from colloidal dispersions. $J$. Chem. Phys. 84:5102-5107.

Livesey, A. K., M. Delaye, P. Licinio, and J. C. Brochon. 1987. Maximum entropy analysis of dynamic parameters via the Laplace transform. Faraday Discuss. Chem. Soc. 83: 1-12.

McWhirter, J. G., and E. R. Pike. 1978. On the numerical inversion of the Laplace transform and Fredholm integral equations of the first kind. $J$. Phys. A. Math. Gen. 11:1729-1745.

Privat, J. P., Ph. Wahl, and J. C. Brochon. 1985. A new method for decay-associated fluorescence spectroscopy. Application to the tryptophan zwitterion. Biochimie (Paris). 67:949-958.

Robbins, R. J., G. R. Fleming, G. S. Beddard, G. W. Robinson, P. J. Thistlethwaite, and G. J. Woolfe. 1980. Photophysics of aqueous tryptophan: pH and temperature effects. J. Am. Chem. Soc. 102:62716279.
Scott, P. F. 1981. A 31 gigahertz map of W3[OH] with a resolution of 0.3 arcsec. Mon. Not. R. Astr. Soc. 194:25P-29P.

Shore, J. E., and R. W. Johnson. 1980. Axiomatic derivation of maximum entropy and the principle of minimum cross-entropy. I.E.E.E. (Inst. Electr. Electron. Eng.) Trans. Biomed. Eng. IT26:26-37.

Sibisi, S. 1983a. Ill conditioned inverse problems. Ph.D. Thesis, University of Cambridge.

Sibisi, S. 1983b. Two-dimensional reconstructions from one-dimensional data by maximum entropy. Nature (Lond.). 301:134-136.

Skilling, J., and R. K. Bryan. 1984. Maximum entropy image reconstruction: general algorithm. Mon. Not. R. Astr. Soc. 211:111-124.

Szabo, At. 1984. Theory of fluorescence depolarisation in macromolecules and membranes. J. Chem. Phys. 81:150-167.

Szabo, A. G., and D. M. Rayner. 1980. Fluorescence decay of tryptophan conformers in aqueous solution. J. Am. Chem. Soc. 102:554-563.

Tikhonov, A., and V. Arsenin. 1977. Solutions of Ill-posed Problems. John Wiley \& Sons, Inc., London.

Wahl, P. H., J. C. Auchet, and B. Donzel. 1974. The wavelength dependence of the response of a pulse fluorometer using the single photoelectron counting method. Rev. Sci. Instrum. 45:28-32.

Wahl, P. H. 1975. Nanosecond pulsefluorimetry. In New Techniques in Biophysics and Cell Biology. Vol. 2. M. Pain and B. Smith, editors. John Wiley \& Sons, Inc., London. 233-285.

Wahl, P. H. 1979. Analysis of fluorescence anisotropy decay by least squares method. Biophys. Chem. 10:91-104.

Wahl, P. H., 1983. Time-resolved fluorescence spectroscopy in biochemistry and biology. NATO ASI (Adv. Sci. Inst.) Ser. A. Life Sci. 69:497-521.

Ware, W. R. 1971. Creation and Detection of the Excited State. Vol. 1. A. A. Lamola, editor. Marcel Dekker, New York. 213-302.

Ware, W. R. 1983. Time-resolved fluorescence spectroscopy in biochemistry and biology. NATO ASI (Adv. Sci. Inst.) Ser. A. Life Sci. 69:299-317.

Yguerabide, J. 1972. Nanosecond fluorescence spectroscopy of macromolecules. Methods Enzymol. 26:498-578.

Zuker, M., A. G. Szabo, L. Bramall, D. T. Krajcarski, and B. Selinger. 1985. Delta function convolution method (DFCM) for fluorescence decay experiments. Rev. Sci. Instrum. 56:14-22. 